\title{
Spatial anisotropy of the exchange integrals in Mn-doped wurtzite-type semiconductors
}

\author{
R. O. Kuzian, ${ }^{1}$ A. M. Daré, ${ }^{2}$ A. Savoyant, ${ }^{2}$ S. D'Ambrosio, ${ }^{2}$ and A. Stepanov ${ }^{2}$ \\ ${ }^{1}$ Institute for Materials Science, Krzhizhanovskogo 3, Kiev 03180, Ukraine \\ ${ }^{2}$ IM2NP, CNRS UMR 6242, FST, Aix-Marseille Université, F-13397 Marseille Cedex 20, France \\ (Received 9 August 2011; revised manuscript received 26 September 2011; published 20 October 2011)
}

\begin{abstract}
We propose an alternative explanation, as compared to one reported in the literature, to the experimentally observed spatial anisotropy $\xi=\Delta J_{1} / J_{1}^{\text {in }}\left(\Delta J_{1}=J_{1}^{\text {in }}-J_{1}^{\text {out }}\right)$ of the nearest neighbor exchange integral $J_{1}$ in Mndoped semiconductors with the wurtzite structure. We show that the main contribution to $\xi$ is ferromagnetic and comes from a looped exchange path which involves two distinct anions. A comparison between our calculations, that use the results of photoemission spectroscopy as input parameters, and the available data for three wurtzite materials, CdS:Mn, CdSe:Mn, and $\mathrm{ZnO}: \mathrm{Mn}$, shows a good quantitative agreement between theory and experiment in the case of cadmium compounds and only a qualitative one in the case of $\mathrm{ZnO}: \mathrm{Mn}$.
\end{abstract}

DOI: 10.1103/PhysRevB.84.165207

PACS number(s): 75.30.Et

\section{INTRODUCTION}

Diluted magnetic semiconductors (DMSs), semiconductors in which the transition-metal ions (TMIs) substitute diamagnetic cations, have become particularly attractive as promising materials for spin electronics. ${ }^{1,2}$ Clear progress has been achieved in understanding of their magnetic properties; however there is still a lot more work to be done in order to clarify the underlying mechanisms behind the spin-spin interactions between TMIs. ${ }^{3}$

It is well established that without an additional doping the magnetic properties of DMSs are dominated by the $d-d$ antiferromagnetic (AFM) superexchange interactions, ${ }^{4}$ characterized by the exchange coupling $J_{d d}$.

Anderson proposed an attractive microscopic picture of the superexchange in magnetic insulators based on a perturbative treatment of the hybridization process. ${ }^{5}$ Its straightforward application to DMS physics was done more than 20 years ago by Larson, Hass, Ehrenreich, and Carlsson in their widely referenced paper, treating II-VI zinc-blende compounds, such as $\mathrm{Mn}$-doped tellurides, selenides, and sulfides. ${ }^{6}$

Since then, wide-gap materials (i.e., GaN:Mn, ZnO:Mn, etc.) with the wurtzite structure became the focus of extensive research that generated a large body of experimental data from photoemission (PE) spectroscopy ${ }^{7-14}$ and magnetic properties measurements. ${ }^{15-20}$ Importantly, the results of magnetization step experiments on wurtzite DMSs reveal that two the next nearest neighbour (NN) $J_{d d}$ parameters are required for their description (which in II-VI DMSs are denoted by $J_{1}^{\text {in }}$ and $J_{1}^{\text {out }}$ ) in contrast with zinc-blende materials where only one $J_{1}$ is sufficient. ${ }^{19,20}$

Although, some attempts were made to gain a better understanding of the exchange interaction in wurtzite materials, there still remains several unsolved problems. As we show in the present paper, the available theoretical estimates fail to account for the experimentally observed spatial anisotropy of $J_{1}, \xi=\Delta J_{1} / J_{1}^{\text {in }}\left(\Delta J_{1}=J_{1}^{\text {in }}-J_{1}^{\text {out }}\right)$; therefore, a detailed analysis of the superexchange mechanism is indispensable. Moreover, it is of fundamental importance to relate this analysis to the PE data, offering the most valuable information on the $s, p-d$ hybridization.

In this paper we will address these points by developing a simple model for the NN exchange parameters in Mn-doped
DMSs, that is essentially based on the theoretical approach used for the treatment of PE experiments.

\section{THEORETICAL MODEL}

We consider a pair of TMIs and several ligand ions between them within the $p-d$ model. The Hamiltonian is

$$
\hat{H}=\hat{H}_{d}+\hat{H}_{p}+\hat{T}_{p d},
$$

where $\hat{H}_{d}$ and $\hat{H}_{p}$ are on-site Hamiltonians for TMIs and ligands, respectively, and $\hat{T}_{p d}$ describes electron hoppings between TMIs and ligands.

In the single ion Hamiltonian we include the diagonal oneparticle terms and dominant Coulomb interactions

$$
\begin{aligned}
\hat{H}_{d}= & \epsilon_{d} \hat{N}_{d}+\hat{H}_{C F, d}+\hat{W}, \\
\hat{W}= & U_{d} \sum_{m} \hat{n}_{m, \uparrow} \hat{n}_{m, \downarrow}+\frac{U_{d}^{\prime}}{2} \sum_{m \neq m^{\prime}} \hat{n}_{m} \hat{n}_{m^{\prime}} \\
& -\frac{J_{H d}}{2} \sum_{m \neq m^{\prime}}\left(2 \mathbf{s}_{m} \mathbf{s}_{m^{\prime}}+\frac{1}{2} \hat{n}_{m} \hat{n}_{m^{\prime}}\right) \\
& +J_{H d} \sum_{m \neq m^{\prime}} d_{m, \uparrow}^{\dagger} d_{m^{\prime}, \uparrow} d_{m, \downarrow}^{\dagger} d_{m^{\prime}, \downarrow}, \\
\hat{H}_{p}= & \epsilon_{p} \hat{N}_{p}+\frac{U_{p}}{2}\left(\hat{N}_{p}^{2}-\hat{N}_{p}\right)+\hat{H}_{C F, p},
\end{aligned}
$$

where

$$
\begin{gathered}
\hat{N}_{l} \equiv \sum_{m} \hat{n}_{m}, \hat{n}_{m} \equiv \sum_{s} \hat{n}_{m, s}, \\
\hat{n}_{m, s}=c_{m, s}^{\dagger} c_{m, s}, \\
\hat{H}_{C F}=\sum_{m, s} V_{m m} \hat{n}_{m, s} \\
U_{d}=A+4 B+3 C, \\
J_{H d}=\frac{5}{2} B+C, \\
U_{d}^{\prime}=U_{d}-2 J_{H d}=A-B+C,
\end{gathered}
$$

$\epsilon_{d}, \epsilon_{p}$ are the one-particle energies of $d$ and $p$ states, $A, B, C$ are the Racah's parameters of TMIs, $U_{p}$ is the Coulomb repulsion 
on oxygen and $\hat{H}_{C F}$ is the crystal field (CF) Hamiltonian. It is convenient to use the hole notations; then the operator $c_{m, s}^{\dagger}=d_{\alpha, m, s}^{\dagger}\left(p_{\beta, m, s}^{\dagger}\right)$ creates a hole with the one-particle basis $d(p)$ wave function and spin projections $s$ on TMI (numbered by $\alpha=1,2$ ) and ligand sites (numbered by $\beta=1,2$ ), respectively; $m=x, y, z, v, w\left(m=x y, y z, z x, x^{2}-y^{2}, 3 z^{2}-r^{2}\right)$ for the TMI in trigonal (tetragonal) axes, and $n=x, y, z$ for the ligand. In the ground state of $H_{l}$, the $d$ shell of the TMI contains $N$ holes, and the ligand has the closed $p$ shell with $n_{p}=6$ electrons (zero holes).

The hopping Hamiltonian

$$
\hat{T}_{p d}=\sum_{n, m, \alpha, \beta, s} t_{\alpha, m, \beta, n}\left(d_{\alpha, m, s}^{\dagger} p_{\beta, n, s}+p_{\beta, n, s}^{\dagger} d_{\alpha, m, s}\right)
$$

couples configurations with different numbers of $d$ and $p$ holes. We will account for the coupling between the configurations $d^{N} p^{0} d^{N}, d^{N-1} p^{1} d^{N}, d^{N-1} p^{2} d^{N-1}$, and $d^{N-1} p^{0} d^{N+1}$.

The Hamiltonian (2) was introduced by Kanamori, and it was used in the form written for electrons for an analysis of the $2 p$ core-level PE spectra of transition metal compounds in Refs. 8 and 21 and in other papers of this group. We will use the approximate Hamiltonian (2), with its parameters determined from PE experiments. The electron-hole transform for the basis of real functions is $a_{m,-s}^{\dagger} \equiv c_{m, s}\left(a_{m,-s}^{\dagger}\right.$ creates an electron). It does not change the operator form of the Hamiltonian, and parameters of the interaction, but for the one-particle parameters we have for the electrons

$$
\begin{gathered}
\epsilon_{p, \mathrm{el}}=-\left[\epsilon_{p}+U_{p}\left(2 R_{p}-1\right)\right], \\
\epsilon_{d, \mathrm{el}}=-\left[\epsilon_{d}+U_{d}+\left(2 U_{d}^{\prime}-J_{H d}\right)\left(R_{d}-1\right)\right],
\end{gathered}
$$

where $R_{l}=(2 l+1)$. The hopping term simply changes the sign. Bocquet et al. ${ }^{21}$ neglected the Coulomb repulsion on the ligand $U_{p} \approx 0$. They introduced two additional parameters

$$
\begin{aligned}
U=U_{d} & -\frac{20}{9} J_{H d}=A-\frac{14}{9} B+\frac{7}{9} C, \\
\Delta & =\epsilon_{d, \mathrm{el}}-\epsilon_{p, \mathrm{el}}+U N_{e l} \\
& =\epsilon_{p}-\epsilon_{d}-U(N-1),
\end{aligned}
$$

where $N_{e l}=10-N$ is the number of electrons in the ground state of the TMI. Note two important misprints in the bottom of Table II of Ref. 21, the first one concerns the expression for $U$, and the second one is in the expression for the center of gravity of the $d^{N_{e l}}$ multiplet, which should be $E\left(d^{N_{e l}}\right)=$ $U N_{e l}\left(N_{e l}-1\right) / 2$.

The Hund exchange fixes the ground state of the TMI. We assume that the TMI is in the high-spin state (i.e., $S=5 / 2$ for $\mathrm{Mn}^{2+}$ ).

The interaction term in (2) may be written as

$$
\begin{aligned}
\hat{W}= & U_{d} \sum_{m} \hat{n}_{m, \uparrow} \hat{n}_{m, \downarrow} \\
& +\left(U_{d}^{\prime}-\frac{J_{H d}}{2}\right)\left[\frac{\hat{N}_{d}^{2}-\hat{N}_{d}}{2}-\sum_{m} \hat{n}_{m, \uparrow} \hat{n}_{m, \downarrow}\right] \\
& -J_{H d}\left(\hat{\mathbf{S}}^{2}-\frac{3}{4} \hat{N}_{d}+\frac{3}{2} \sum_{m} \hat{n}_{m, \uparrow} \hat{n}_{m, \downarrow}\right) \\
& +J_{H d} \sum_{m \neq m^{\prime}} d_{m, \uparrow}^{\dagger} d_{m^{\prime}, \uparrow} d_{m, \downarrow}^{\dagger} d_{m^{\prime}, \downarrow} .
\end{aligned}
$$

Then, neglecting the $d$-state CF splitting compared to $\Delta$, we have for $N$ holes in the $d$ shell [cf. Eq. (3) of Ref. 21]:

$$
\begin{aligned}
\left\langle S, S, N\left|\hat{H}_{d}\right| S, S, N\right\rangle & =\epsilon_{d} N+U_{d} p+\left(U_{d}^{\prime}-\frac{J_{H d}}{2}\right)\left[\frac{1}{2} N(N-1)-p\right]-\frac{J_{H d}}{4} n_{1}\left(n_{1}-1\right) \\
& =\epsilon_{d} N+U_{d} p+U_{d}^{\prime}\left[\frac{1}{2} N(N-1)-p\right]-\frac{J_{H d}}{2}\left[N_{\uparrow}\left(N_{\uparrow}-1\right)+N_{\downarrow}\left(N_{\downarrow}-1\right)\right] \equiv E(S, N),
\end{aligned}
$$

with $p=N-5$ being the number of $d$-hole pairs in the same orbital ( $p=0$ for $N \leqslant 5), n_{1}=N-2 p=N_{\uparrow}-N_{\downarrow}=2 S$ is the number of singly occupied orbitals, and $N_{\uparrow, \downarrow}$ is the number of holes with a given spin projection index.

We write the magnetic interaction between two TMIs as $H_{J}=-2 J S_{1} S_{2}$. We calculate the superexchange parameter using the resolvent method ${ }^{22}$ which in the fourth order gives the formula ${ }^{6,23}$

$$
\begin{aligned}
J= & -\frac{1}{2 \sqrt{S_{1} S_{2}}} \\
& \times \sum_{I_{1}, I_{2}, I_{3}} \frac{\left\langle f\left|\hat{T}_{p d}\right| I_{1}\right\rangle\left\langle I_{1}\left|\hat{T}_{p d}\right| I_{2}\right\rangle\left\langle I_{2}\left|\hat{T}_{p d}\right| I_{3}\right\rangle\left\langle I_{3}\left|\hat{T}_{p d}\right| i\right\rangle}{\left(E_{0}-E_{I_{1}}\right)\left(E_{0}-E_{I_{2}}\right)\left(E_{0}-E_{I_{3}}\right)},
\end{aligned}
$$

where the $|i\rangle(|f\rangle)$ denotes the initial (final) state, represented by a ket vector $|S, S-1\rangle(|S-1, S\rangle)$, and $\left|I_{i}\right\rangle$ are the intermediate states. Here the notation $\left|M_{1}, M_{2}\right\rangle$ means that the first TMI has the spin projection $M_{1}$, and the second one has its spin projection $M_{2}$. After tedious but straightforward algebra, we obtain ${ }^{24}$

$$
J=-\frac{1}{2 S^{2} \Delta_{\text {eff }}^{2}} \sum_{\beta, \beta^{\prime}}\left(\frac{r^{2}}{U_{\text {eff }}}+\frac{2}{2 \Delta_{\text {eff }}+U_{p} \delta_{\beta \beta^{\prime}}}\right) E_{\beta \beta^{\prime}},
$$

where

$$
E_{\beta \beta^{\prime}}=\sum_{m, m^{\prime}, n, n^{\prime}}^{o c c} t_{1, m, \beta, n} t_{2, m^{\prime}, \beta, n} t_{1, m, \beta^{\prime}, n^{\prime}} t_{2, m^{\prime}, \beta^{\prime}, n^{\prime}} .
$$

Summation in Eq. (10) goes over orbitals occupied by holes in the TMI ground state.

The first term in parentheses of Eq. (9) corresponds to Anderson's processes ${ }^{5}$ where a hole is transferred from one TMI to another. The energy denominator for these processes 
is

$$
\begin{aligned}
U_{\mathrm{eff}} & \equiv\left(E_{0}-E_{I_{2}}\right) \\
& =E^{*}(N+1)+E(S-1, N-1)-2 E(S, N) \\
& =U+J_{H d}\left(N+\frac{11}{9}-2 p\right),
\end{aligned}
$$

with $E^{*}(N+1)=\epsilon_{d}+U_{d}+U_{d}^{\prime}(N-1)-J_{H d} p+E(S, N)$ being the energy of the intermediate state $d_{m, \downarrow}^{\dagger} \hat{n}_{m, \uparrow}|S, S, N\rangle$, which occurs when to a TMI in a high spin state, with all the spins up, a hole with its spin down is added. Note the large contribution of Hund's exchange to $U_{\text {eff }}$ for many-electron TMI states $\Delta U_{H d}=J_{H d}(N-1-2 p)$. This renormalization of the effective Hubbard repulsion $U$ was originally introduced for the description of PE experiments. ${ }^{7}$ As far as we know, it was not previously discussed in connection with the superexchange model of ionic solids. $\Delta U_{H d}$ vanishes for the $d^{9}$ configuration $\left(N=1, p=0\right.$, e.g., $\mathrm{Cu}^{2+}$ ions, which are responsible for the magnetic properties in cuprates) and for the $d^{1}$ configuration $(N=9, p=4)$, but it reaches its maximum value of $4 J_{H d}$ for the $d^{5}$ configuration of the $\mathrm{Mn}^{2+}$ ion.

The second term in the parentheses of Eq. (9) comes from the processes having the intermediate state $\left|I_{2}\right\rangle$, which arises either when two holes occupy the same ligand or when they occupy two different bridging ligands. ${ }^{6,23}$ In this case, the denominators contain an effective charge transfer energy

$$
\Delta_{\mathrm{eff}}=\Delta+J_{H d}\left[\frac{7}{9}(N-1)-p\right],
$$

which also depends on $J_{H d}$ for a many-electron TMI state. ${ }^{7}$

The hopping integrals $t_{\alpha, m, \beta, n}{ }^{25}$ between the $m$ th $d$ function of the metal ion and the $n$th $p$ function of the ligand are expressed via direction cosines $l, m, n$ of the vector $\mathbf{R}_{\beta}-\mathbf{R}_{\alpha}$, and two Slater-Koster parameters ${ }^{26} V_{p d \sigma}$ and $V_{p d \pi}$, which depend on the distance $R=\left|\mathbf{R}_{\beta}-\mathbf{R}_{\alpha}\right|$ and for which we use the notation currently adopted in the PE literature, $V_{p d \sigma} \equiv(p d \sigma)$ and $V_{p d \pi} \equiv(p d \pi)$. Harrison's parametrization is applied for $(\mathrm{pdm})$ parameters in the case of hopping between Mn ions and distant anions: $:^{27}$

$$
(p d m)(R)=\eta_{p d m} \frac{\hbar^{2} r_{d}^{3 / 2}}{m R^{7 / 2}},
$$

where $\eta_{p d \sigma}=-2.95, \eta_{p d \sigma} / \eta_{p d \pi}=-2.16$, and the value $r_{d}=0.925 \AA$ for the Mn ion. We also take into account the dependence of the hopping integrals on the $d$-shell filling by introducing a reduction coefficient in the term $\propto 1 / U_{\text {eff }}$ in Eq. (9), ${ }^{23}$ i.e., $r=0.8$.

For $\mathrm{Mn}^{2+}\left(d^{5}\right)$ Eqs. (11) and (12) give ${ }^{11}$

$$
\begin{aligned}
& U_{\text {eff }}=U+(140 B+56 C) / 9, \\
& \Delta_{\text {eff }}=\Delta+28 J_{H d} / 9=\Delta+(70 B+28 C) / 9,
\end{aligned}
$$

where $B=0.119 \mathrm{eV}, C=0.412 \mathrm{eV}^{28}$ and we assume that $U_{p}=0$.

\section{RESULTS AND DISCUSSION}

In order to facilitate the ensuing discussion, in Fig. 1 we present the TMI sites and their environment in the wurtzite crystal structure, using as an example a Mn-doped ZnO system.

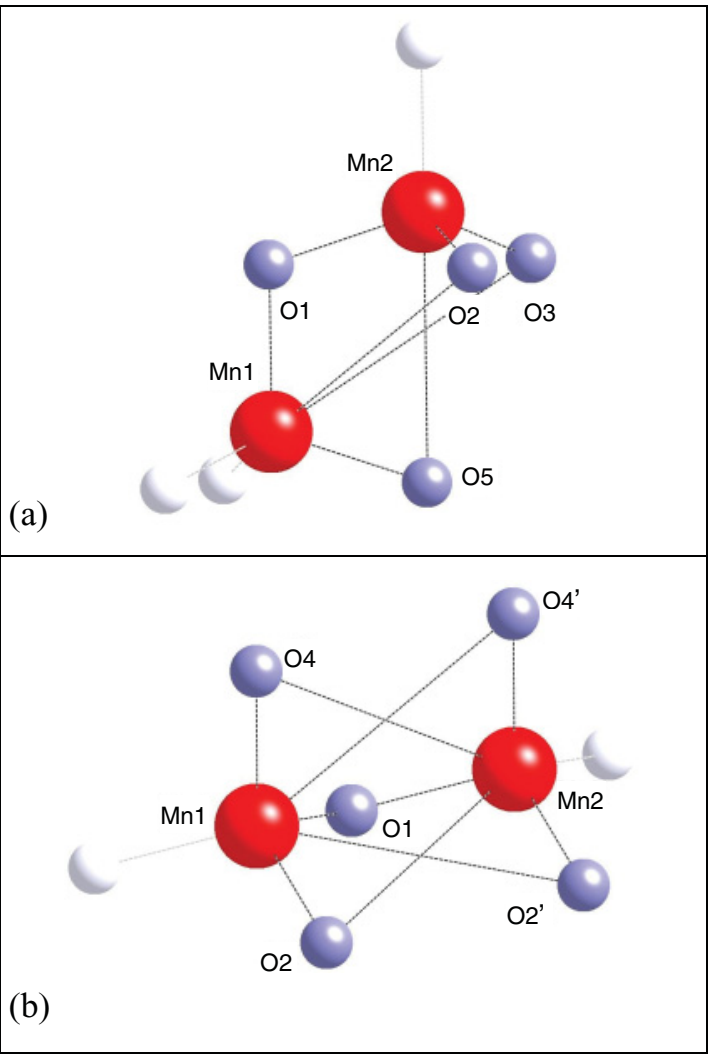

FIG. 1. (Color online) Fragments of $\mathrm{ZnO}: \mathrm{Mn}$ crystal structure presenting the positions of two substitutional nearest neighbor $\mathrm{Mn}$ ions and their oxygen surroundings: (a) $\mathrm{Mn}$ and $\mathrm{O}$ ions involved in $J_{1}^{\text {out }}$ exchange integral calculations are shown; (b) the same for $J_{1}^{\text {in }}$ calculations.

Two groups of NN cation sites have to be distinguished in the wurtzite lattice: ${ }^{18}$ six "in-plane" NNs which are in the same $a b$ plane as the central cation (the corresponding exchange constant labelled $J_{1}^{\text {in }}$ ) and six “out-of-plane” NNs which are coupled to the central cation by $J_{1}^{\text {out }}$. In addition, each cation is surrounded by a number of anions which in the superexchange picture are involved in hopping processes. In the wurtzite lattice the atomic positions are determined by three lattice parameters $a, c$, and $u .^{29}$ Knowing them, one can obtain the relative positions of a given cation and surrounding anions and in this way evaluate the exchange contributions from different exchange paths using our Eq. (9). We will denote by $J_{\alpha \beta}$ an exchange integral corresponding to a 4th order hopping process via $\mathrm{A}_{\alpha}$ and $\mathrm{A}_{\beta}$ anions which can be schematically presented as $\mathrm{Mn}_{1}-\mathrm{A}_{\alpha}-\mathrm{Mn}_{2}-\mathrm{A}_{\beta}-\mathrm{Mn}_{1}$.

\section{A. Earlier model}

We are now in a position to discuss the origin of the observed anisotropy of $J_{1}$. First, we recall the currently available explanation of $\xi$ which is the following. ${ }^{30}$ Since, in the ideal wurtzite structure the virtual hopping between two TMIs via $\mathrm{NN}$ anions [in both Figs. 1(a) and 1(b) these anions are labeled as $\mathrm{O}_{1}$ ] is the same for in-plane and out-of-plane processes, the calculations give $J_{11}($ in $)=J_{11}$ (out), i.e., $\Delta J_{1}=0$ and $\xi=0$. In order to have $\xi \neq 0$, one should take into account the hopping processes via more distant anions. An examination of 
Fig. 1(a) shows that for the out-of-plane geometry there exist three additional exchange paths via the oxygens labeled $\mathrm{O}_{2}$, $\mathrm{O}_{3}$, and $\mathrm{O}_{5}$, which lead to $J_{22}, J_{33}$, and $J_{55}$, respectively. While, as it is clear from Fig. 1(b), for the in-plane geometry, one can find four such exchange paths via the oxygens labeled as $\mathrm{O}_{2}$, $\mathrm{O}_{2^{\prime}}, \mathrm{O}_{4}$, and $\mathrm{O}_{4^{\prime}}$. Then, if one supposes that all these additional processes via distant anions are more or less equivalent and contribute antiferromagnetically to $J_{1}$, it follows that $J_{1}^{\text {in }}$ will gain from one more of such contributions; hence $\left|J_{1}^{\text {in }}\right|>$ $\left|J_{1}^{\text {out }}\right|$ in this model. A numerical estimation gives for CdS:Mn $\xi=0.16$ close to $\xi=0.13$ inferred from the magnetization step experiment. ${ }^{19}$

This rough estimate should be considered, however, with some care, as our accurate calculations show that the exchange integrals, such as $J_{22}, J_{33}$, etc., contribute only a very small part to $J_{1}$. In fact, the ratio $J_{\alpha \alpha} / J_{i n}$ (for $\alpha=2,2^{\prime}, 3$, and 4) is found to be about 0.01 ; hence the above estimate of $\xi$ should be scaled down by an order of magnitude.

\section{B. Input parameters}

In Table I the results of our analysis are shown for three wurtzite compounds. The upper part of the table lists the input parameters, and its lower part shows the obtained results in a comparison with the experimental data from magnetization step measurements, which are presented in parentheses.

First, we explain our choice of the input parameters. In the case of CdS:Mn and CdSe:Mn for which no detailed PE data are available, the results of the PE experiments for similar compounds, such as ZnS:Mn and ZnSe:Mn, respectively, have been taken. Then, $U$ and $\Delta$ parameters were used unchanged in

TABLE I. Comparison of the calculated and experimental exchange coupling constants for three wurtzite compounds. The electronic structure parameters are in $\mathrm{eV}$, and the exchange integrals are in Kelvins. The experimental data from magnetization step measurements are presented in parentheses.

\begin{tabular}{lccc}
\hline \hline Parameter & CdSe:Mn & CdS:Mn & ZnO:Mn \\
\hline$a(\AA)$ & $4.2999^{\mathrm{a}}$ & $4.1367^{\mathrm{b}}$ & $3.252^{\mathrm{c}}$ \\
$c / a$ & $1.6305^{\mathrm{a}}$ & $1.6235^{\mathrm{b}}$ & $1.600^{\mathrm{c}}$ \\
$u$ & $0.376^{\mathrm{d}}$ & $0.377^{\mathrm{d}}$ & $0.383^{\mathrm{c}}$ \\
$U$ & $4^{\mathrm{e}}$ & $4^{\mathrm{e}}$ & $5^{\mathrm{f}}$ \\
$\Delta$ & $2^{\mathrm{e}}$ & $3^{\mathrm{e}}$ & 4.7 \\
$(p d \sigma)$ & -0.98 & -1.22 & -1.8 \\
\hline$J_{1}^{\text {in }}$ & $-8.1\left(-8.1^{\mathrm{g}}\right)$ & $-11.1\left(-11.0^{\mathrm{h}}\right)$ & $-24.0\left(-24.3^{\mathrm{i}}\right)$ \\
$J_{1}^{\text {out }}$ & $-7.0\left(-7.0^{\mathrm{g}}\right)$ & $-9.4\left(-9.6^{\mathrm{h}}\right)$ & $-18.7\left(-18.2^{\mathrm{i}}\right)$ \\
$J_{11}$ & -8.0 & -10.8 & -21.7 \\
$J_{55}$ & -0.2 & -0.3 & -0.8 \\
$J_{15}$ & 1.2 & 1.7 & 3.7 \\
$\Delta J_{1} / J_{1}^{\text {in }}$ & $0.14(0.14)$ & $0.15(0.13)$ & $0.22(0.25)$ \\
\hline \hline
\end{tabular}

${ }^{\mathrm{a}}$ Reference 31 .

${ }^{\mathrm{b}}$ Reference 32 .

${ }^{\mathrm{c}}$ References 29.

${ }^{\mathrm{d}}$ Reference 33.

${ }^{\mathrm{e}}$ References 7 and 11.

${ }^{\mathrm{f}}$ References 11,36 , and 37 .

${ }^{\mathrm{g}}$ Reference 15,16 , and 17.

${ }^{\mathrm{h}}$ References 18 and 19.

${ }^{\mathrm{i}}$ Reference 20. the fitting, and ( $p d \sigma)$ was slightly reduced from their original values in zinc compounds $[(p d \sigma)=-1.3 \mathrm{eV}$ for $\mathrm{ZnS}: \mathrm{Mn}$ and $(p d \sigma)=-1.1 \mathrm{eV}$ for ZnSe:Mn] in order to account for the larger anion-cation distance in cadmium materials as compared to zinc ones and to have the best fit of the experimental data.

In the case of $\mathrm{ZnO}: \mathrm{Mn}$ the choice of the electronic structure parameters is somewhat more delicate. The only paper which reports both the $\mathrm{PE}$ data and their analysis, in the framework of the configuration interaction model, is Ref. 11. According to this study $U=5.2 \pm 1 \mathrm{eV}, \Delta=6.5 \pm 1 \mathrm{eV}$, and $(p d \sigma)=$ $-1.6 \pm 0.2 \mathrm{eV}$. By putting these parameters into our Eq. (9), we get $J_{1}^{\text {in }} \simeq-8 \mathrm{~K}$ and $J_{1}^{\text {out }} \simeq-6.3 \mathrm{~K}$ (Ref. 34 ), both of which are too small by a factor of approximately three, as compared to the data. The reason of this discrepancy is not clear for the present. A plausible explanation may come from the recent PE results ${ }^{35}$ which reveal the presence of occupied $\mathrm{Mn}^{2+}$ levels above the valence-band edge of $\mathrm{ZnO}$, whereas the analysis in Ref. 11 was made assuming the position of these levels below the valence-band edge. In this situation, we are led to use additional information regarding the electronic structure parameters of $\mathrm{ZnO}: \mathrm{Mn}$. In our fitting procedure we assumed $U=5 \mathrm{eV}$, a typical value of $U$ used in the local spin density approximation $+\mathrm{U}$ calculations of $\mathrm{ZnO}: \mathrm{Mn},{ }^{36,37}$ that is close to the PE data. Next, the hopping parameter $(p d \sigma)$ was taken to be $-1.8 \mathrm{eV}$, according to Harrison's formula [Eq. (13)] and within the stated uncertainty of the PE data. As far as the value of $\Delta=6.5 \mathrm{eV}$ is concerned, even the authors of Ref. 11 consider it somewhat overestimated, and for their evaluation of the $J_{p d}$ integral they use the value of $5.5 \mathrm{eV}$ leading to $J_{p d}=-2.7 \mathrm{eV}$, which is still very far from $J_{p d}=0.1$ to $0.5 \pm 0.2 \mathrm{eV}$, obtained from the magneto-optical data. ${ }^{38,39}$ Therefore, we decided not to fix $\Delta$, but rather use it as a fitting parameter.

\section{Comparison with experiment}

Some comments should be made regarding the results presented in Table I. We have analyzed in detail various hopping processes via anions located at the distances up to $\sqrt{19 / 8} a$ from $\mathrm{Mn}$ ions. However, the contributions from these distant exchange paths yield only small corrections to the absolute value of $J_{1}^{\text {in }}$ and $J_{1}^{\text {out }}$, and do not change much the $\xi$ value. This is why $J_{1}^{\text {in }}$ in Table I is represented by the $J_{11}$ integral, i.e., $J_{1}^{\text {in }}=J_{11}$. In a similar way $J_{1}^{\text {out }}$ in Table I contains only main corrections due to the next-nearest neighbour anion, i.e., $J_{1}^{\text {out }}=J_{11}+J_{55}+J_{15}$.

Our important finding is that the main contribution to $\xi$ comes from $J_{15}$, a looped exchange path which includes two distinct anions. This ferromagnetic looped process contributes to $J_{1}^{\text {out }}$, but does not contribute to $J_{1}^{\text {in }}$. The strongest AFM exchange coupling in both geometries is given by $J_{11}$ and a weak one by $J_{55}$.

As it can be seen from Table I, the obtained results for cadmium compounds are in a good quantitative agreement with the experimental data, and this is with practically no adjustable parameters. In both cases the principal contribution to $\xi$ comes from the ferromagnetic looped exchange $J_{15}$, while $J_{11}($ in $) \simeq J_{11}$ (out), because $\mathrm{CdS}$ and $\mathrm{CdSe}$ both have almost ideal wurtzite structure with $c / a$ close to $\sqrt{8 / 3}$. A slight discrepancy in $\xi$ between the experimental and calculated values may originate from anisotropic parts of 
the exchange coupling (symmetric or antisymmetric, i.e., the Dzyaloshinskii-Moriya term) which are ignored in the present model. Note also, that in the early model calculations ${ }^{6}$ the value of $J_{1}$ for $\mathrm{ZnS}: \mathrm{Mn}$ is nearly twice as large as the corresponding experimental value that was obtained.

With our choice of the input parameters for $\mathrm{ZnO}: \mathrm{Mn}$, we obtain the best fit for the experimental data with $\Delta=4.7 \mathrm{eV}$. In this case, in addition to the $J_{15}$ contribution which is still the dominant one, $\xi$ contains also an important contribution from $J_{11}$, because $J_{11}($ in $)=-24 \mathrm{~K}$, but $J_{11}$ (out) $=-21.7 \mathrm{~K}$, which is clearly due to the distorted wurtzite structure of $\mathrm{ZnO}$ $(c / a=1.6)$.

\section{CONCLUSION}

In conclusion, within the $p-d$ hybridization scheme, widely used for the interpretation of PE data, we have developed a model which allows us to describe the experimentally observed anisotropy $\xi$ of NN exchange coupling constants in Mn-doped wurtzite DMSs. We found that the main contribution to $\xi$ is due to a specific ferromagnetic looped exchange process involving two distinct anions. Using the available data from PE spectroscopy as input parameters, we obtain the estimates for $J_{1}^{\text {in }}$ and $J_{1}^{\text {out }}$, which are in a good agreement with the experimental results in the case of CdSe:Mn and CdS:Mn, but not in the case of $\mathrm{ZnO}: \mathrm{Mn}$. We speculate that, experimentally, this disagreement is due to the midgap $d$ states, recently found in the PE experiments on $\mathrm{ZnO}: \mathrm{Mn}$, however, which was not considered in the earlier PE work. Nevertheless, our study allows us to make some predictions concerning the electronic structure parameters of this material. If one fixes $(p d \sigma)$ in the range of -1.6 to $-1.8 \mathrm{eV}$, which is a quite reasonable choice, then, according to our analysis, $U$ and $\Delta$ cannot exceed 4 to $5 \mathrm{eV}$ in order to fit the experimental data. It will be interesting to verify these predictions in future PE experiments. Another important outcome of this work is that it shows how fine details of hybridization physics in DMSs can be revealed by an analysis of their exchange interactions.

\section{ACKNOWLEDGMENTS}

This work has partly supported by the CNRS-NASU program PICS (Contract. No. 4767). Discussions with R. Hayn are gratefully acknowledged.
${ }^{1}$ H. Ohno, Science 281, 951 (1998).

${ }^{2}$ I. Žutić, J. Fabian, and D. Das Sarma, Rev. Mod. Phys. 76, 323 (2004).

${ }^{3}$ T. Dietl, Nat. Mater. 9, 965 (2010).

${ }^{4}$ J. K. Furdyna, J. Appl. Phys. 64, R29 (1988); H. Ohno, Science 291, 840 (2001); T. Dietl, H. Ohno, F. Matsukura, J. Cibert, and D. Ferrand, ibid. 287, 1019 (2000).

${ }^{5}$ P. W. Anderson, Phys. Rev. 115, 2 (1959).

${ }^{6}$ B. E. Larson, K. C. Hass, H. Ehrenreich, and A. E. Carlsson, Phys. Rev. B 37, 4137 (1988).

${ }^{7}$ T. Mizokawa and A. Fujimori, Phys. Rev. B 48, 14150 (1993).

${ }^{8}$ T. Mizokawa and A. Fujimori, Phys. Rev. B 56, 6669 (1997).

${ }^{9}$ J. Okabayashi, A. Kimura, T. Mizokawa, A. Fujimori, T. Hayashi, and M. Tanaka, Phys. Rev. B 59, R2486 (1999).

${ }^{10}$ J. Okabayashi, T. Mizokawa, D. D. Sarma, A. Fujimori, T. Slupinski, A. Oiwa, and H. Munekata, Phys. Rev. B 65, 161203 (2002).

${ }^{11}$ T. Mizokawa, T. Nambu, A. Fujimori, T. Fukumura, and M. Kawasaki, Phys. Rev. B 65, 085209 (2002).

${ }^{12}$ M. Kobayashi, Y. Ishida, J. 1. Hwang, T. Mizokawa, A. Fujimori, K. Mamiya, J. Okamoto, Y. Takeda, T. Okane, Y. Saitoh, Y. Muramatsu, A. Tanaka, H. Saeki, H. Tabata, and T. Kawai, Phys. Rev. B 72, 201201 (2005).

${ }^{13}$ M. Kobayashi, Y. Ishida, J. I. Hwang, Y. Osafune, A. Fujimori, Y. Takeda, T. Okane, Y. Saitoh, K. Kobayashi, H. Saeki, T. Kawai, and H. Tabata, Phys. Rev. B 81, 075204 (2010).

${ }^{14}$ J. I. Hwang, Y. Ishida, M. Kobayashi, H. Hirata, K. Takubo, T. Mizokawa, A. Fujimori, J. Okamoto, K. Mamiya, Y. Saito, Y. Muramatsu, H. Ott, A. Tanaka, T. Kondo, and H. Munekata, Phys. Rev. B 72, 085216 (2005).

${ }^{15}$ E. D. Isaacs, D. Heiman, P. Becla, Y. Shapira, R. Kershaw, K. Dwight, and A. Wold, Phys. Rev. B 38, 8412 (1988).
${ }^{16}$ S. Foner, Y. Shapira, D. Heiman, P. Becla, R. Kershaw, K. Dwight, and A. Wold, Phys. Rev. B 39, 11793 (1989).

${ }^{17}$ V. Bindilatti, T. Q. Vu, Y. Shapira, C. C. Agosta, E. J. McNiff Jr., R. Kershaw, K. Dwight, and A. Wold, Phys. Rev. B 45, 5328 (1992).

${ }^{18}$ Y. Shapira, S. Foner, D. Heiman, P. A. Wolff, and C. R. McIntyre, Solid State Commun. 71, 355 (1989).

${ }^{19}$ Y. Shapira and V. Bindilatti, J. Appl. Phys. 92, 4155 (2002).

${ }^{20}$ X. Gratens, V. Bindilatti, N. F. Oliveira Jr., Y. Shapira, S. Foner, Z. Golacki, T. E. Haas, Phys. Rev. B 69, 125209 (2004).

${ }^{21}$ A. E. Bocquet, T. Mizokawa, T. Saitoh, H. Namatame, and A. Fujimori, Phys. Rev. B 46, 3771 (1992).

${ }^{22}$ P.-O. Lowdin, J. Chem. Phys. 19, 1396 (1951).

${ }^{23}$ B. E. Larson and H. Ehrenreich, Phys. Rev. B 39, 1747 (1989).

${ }^{24}$ R. O. Kuzian, V. V. Laguta, A.-M. Daré, I. V. Kondakova, M. Marysko, L. Raymond, E. P. Garmash, V. N. Pavlikov, A. Tkach, P. M. Vilarinho, and R. Hayn, Europhys. Lett. 92, 17007 (2010).

${ }^{25}$ Our $t_{\beta, n, \alpha, m}$ are equivalent to $E_{n, m}(l, m, n)$ in Slater-Koster notation. ${ }^{26}$

${ }^{26}$ J. C. Slater and G. F. Koster, Phys. Rev. 94, 1498 (1954).

${ }^{27}$ W. A. Harrison, Electronic Structure and the Properties of Solids (Freeman, San Francisco, 1980).

${ }^{28}$ J. S. Griffith, The Theory of Transition-Metal Ions (Cambridge University Press, London, 1961).

${ }^{29}$ E. H. Kisi and M. M. Elcombe, Acta Crystallogr. Sect. C 45, 1867 (1989).

${ }^{30}$ B. E. Larson, J. Appl. Phys. 67, 5240 (1990).

${ }^{31}$ R. R. Reeber, J. Mater. Sci. 11, 590 (1976).

${ }^{32}$ W. R. Cook Jr., J. Am. Ceram. Soc. 51, 518 (1968).

${ }^{33}$ In the case of CdS and CdSe we used $u=\sqrt{3 / 8}(a / c)$. 
${ }^{34}$ Note that from these values of $J_{1}$ one obtains $\xi=0.21$.

${ }^{35}$ C. A. Johnson, K. R. Kittilstved, T. C. Kaspar, T. C. Droubay, S. A. Chambers, G. M. Salley, and D. R. Gamelin, Phys. Rev. B 82, 115202 (2010).

${ }^{36}$ T. Chanier, M. Sargolzaei, I. Opahle, R. Hayn, and K. Koepernik, Phys. Rev. B 73, 134418 (2006).

${ }^{37}$ P. Gopal and N. A. Spaldin, Phys. Rev. B 74, 094418 (2006).
${ }^{38}$ W. Pacuski, J. Suffczyński, P. Osewski, P. Kossacki, A. Golnik, J. A. Gaj, C. Deparis, C. Morhain, E. Chikoidze, Y. Dumont, D. Ferrand, J. Cibert, and T. Dietl, Phys. Rev. B 84, 035214 (2011).

${ }^{39}$ An explanation of the disagreement between $J_{p d}$ inferred from the magneto-optical data and the photoemission experiments can be found in Refs. 40 and 41.

${ }^{40}$ Tomasz Dietl, Phys. Rev. B 77, 085208 (2008).

${ }^{41}$ T. Chanier, F. Virot, and R. Hayn, Phys. Rev. B 79, 205204 (2009). 